\title{
Tracking progress on the SDGs
}

The latest United Nations review leaves no doubt, countries need to step up efforts and act fast if they want to achieve the Global Goals by 2030.

n September 2015, the UN Sustainable
Development Summit adopted the
2030 Agenda including 17 Sustainable Development Goals (SDGs), 169 targets, and a framework to review countries' progress towards the SDGs led by the High-level Political Forum (HLPF) on Sustainable Development. Each year since the adoption of the Goals, the HLPF has met to share experiences about the implementation of the SDGs. The 2018 meeting was held over 9-18 July at the UN headquarters in New York, where an unprecedented 46 countries reported on their progress on the SDGs.

Besides a few success stories, the review process highlighted limited progress on only some of the SDGs and at a very slow pace. But how has progress been measured and evaluated so far? A number of assessment tools have been developed, such as the official 232 SDG indicators (https://go.nature. com/2M8a7Q4), and practices including voluntary country-led review processes. And to complement those efforts, the Sustainable Development Solutions Network, jointly with the Bertelsmann Stiftung foundation, has published annually - starting in 2016 - the SDG Index and Dashboard Report, which, by adopting specific quantitative and visual tools, presents an overview of countries' advances on the SDGs (http://www.sdgindex. org/overview/). In particular, the Report includes the SDG Index that is calculated for each country using the same basket of SDGsrelevant indicators so that country scores can be ranked; the Index delivers a percentage value representing the progress of a country towards meeting the Global Goals. The Report also includes a visual representation of countries' performance on the SDGs, the dashboard, with the aim of helping governments to identify priority areas for intervention.

The 2018 Report covers, for the first time, all 193 UN member states and includes SDG Trend Dashboards that indicate whether a country is on track to achieve a particular goal by 2030 (http://www.sdgindex.org/ reports/2018/). The Report provides the most comprehensive picture of national progress on the SDGs and offers a useful synthesis of what has been achieved so far. Perhaps unsurprisingly, the best-performing nations are Sweden with an SDG Index score of $85 \%$, Denmark 84.6\%, and Finland 83\%, whereas the worst ones are Democratic Republic of Congo

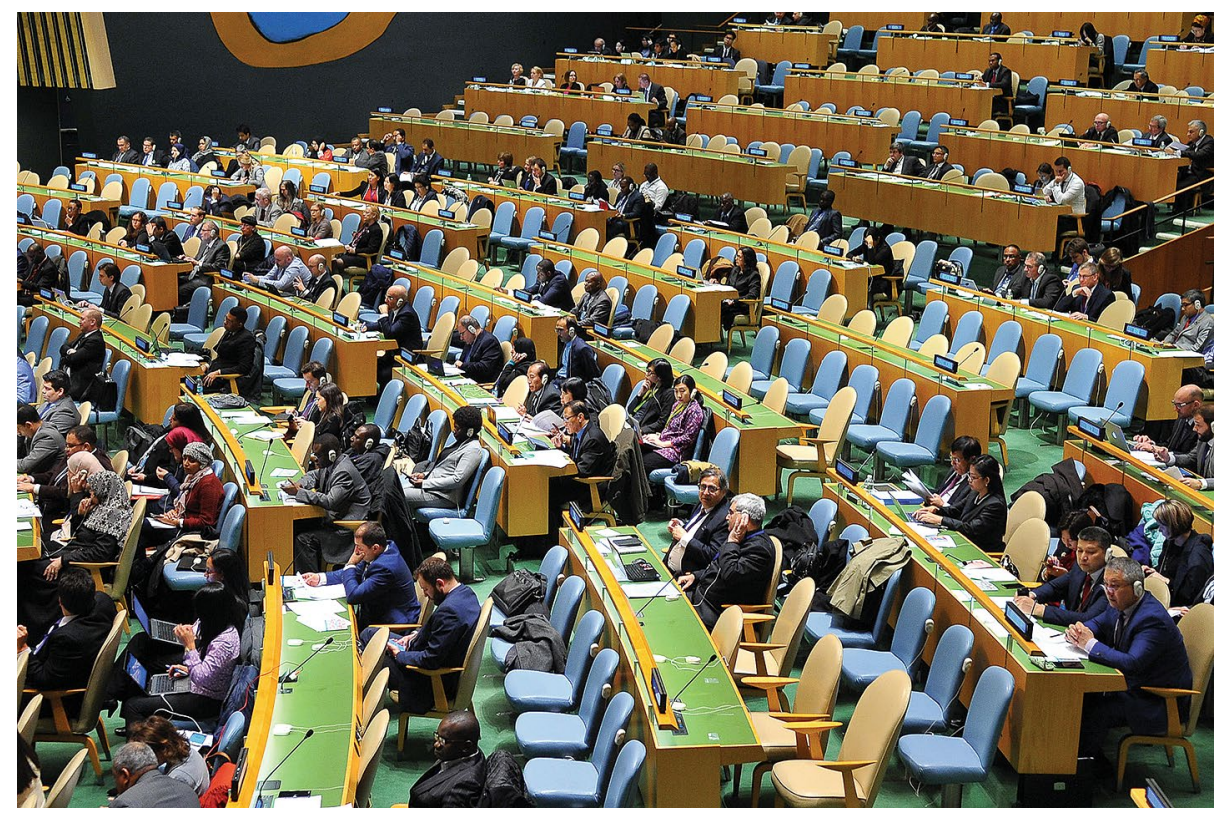

Credit: PACIFIC PRESS / Alamy Stock Photo

with $43.4 \%$, Chad $42.8 \%$ and Central African Republic with only $37.7 \%$. Overall, the scores are well below $100 \%$ for all countries included in the analysis - a disappointing result, especially for the G20 richer economies. In fact, the main message of the Report is worrying: no country is on track to achieve all SDGs by 2030.

Why so? Why is progress so slow? There are many issues at play and the answer is not straightforward. Challenges and opportunities to meet the SDGs vary significantly across different geographical, political and economic contexts. It is crucial to factor in such diversity when taking action and assessing success. But despite the significant efforts discussed above, there is still not enough clarity and confidence about the indicators of progress towards the SDGs and how to use them. A number of the 232 official SDG indicators of the United Nations are still being discussed three years after the launch of the 2030 Agenda (https://unstats.un.org/sdgs/ tierIII-indicators/), something hard to believe given our current technical and statistical abilities. Besides indicators, there is need for new data and innovative approaches to collect them. Most of the data used for example in the SDG Index and Dashboard Reports are from existing databases that were not thought through from a sustainable development perspective. Obviously such existing data sources suffer from gaps on critical SDGs-related targets and yet, at the level of development agencies, there is no clear sign of trying to address the problem.

At the July meeting, the HLPF revealed plans for the next big SDGs-related event: Global Goals Week 2018, which will be held in New York over 22-29 September (https:// go.nature.com $/ 2 \mathrm{n} 0 \mathrm{vl} 7 \mathrm{D}$ ). It is the third annual week of action convened by the UN to include partners from around the globe in support of the SDGs. Our hope is that the event will continue to raise awareness among citizens around the world about the importance of achieving the SDGs on time, and governments will step up efforts and act faster than they have done so far - our window of opportunity to enhance human well-being while safeguarding the health of the planet is rapidly closing down. We need more academic research to inform action and more effective communication channels between academia and decision makers. Ultimately, we need the participation and commitment of civil society and political and industry leaders if we want to successfully implement the 2030 Agenda.

Published online: 14 August 2018 https://doi.org/10.1038/s41893-018-0131-z 